with the collection and analysis of information about patients treated in general hospitals. It is intended to become a "tool of management" for both clinicians and administrators, and the hope is that this information can be provided, with the aid of computers, speedily enough to make it realistic for action at both group and hospital level. Hospital activity analysis, however, can supply neither common sense nor courage.

While it is important to have this information quickly it is even more important that it should be accurate and complete, otherwise wrong conclusions might be drawn from these analyses. Who is to watch over the assembling of this great array of information about patients? Who will ensure that what is written down is both correct and complete? There is only one person who is already trained for and involved in dealing with admissions, discharges, bed-occupancy, diagnostic and operation indexes, and many similar and equally complicated matters of fact; it is the medical records officer-a name now clearly becoming out of date. Only such a person is able to guide and support the computer team when they try to unravel and analyse the manifold procedures of which they themselves have no first-hand experience. Tomorrow's hospitals will realize more and more the need for this experienced person who, with simpler and humbler tools than the computer, has over many years been collecting reasonably good and accurate information about patients and their illnesses.

The hospital activity analyst already exists under the name of medical records officer, and when this sophisticated tool of management known as hospital activity analysis becomes available for "performance monitoring" by clinicians and administrators, the medical records officer will be recognized as an essential member of the hospital administrative team.

The medical records officers have faced up well to their responsibilities. Some might say that they take themselves too seriously; maybe, but they cannot take their work too seriously. They formed an association over 20 years ago. They hold examinations; they confer locally, nationally, and now as a European participant. It would weary the reader to catalogue all their activities.

What is so remarkable is that the medical records officer is still regarded as no more than a cog in the administrative machine. Unlike, say, physiotherapy or radiography this is not a recognized auxiliary profession. One consequence of this is that at the lowest level members of the medical records staff come and go with disconcerting frequency; yet these young women handle confidential documentary material. I find this frightening. A girl wishing to become, for example, a physiotherapist goes through a recognized course of training; she belongs to a profession with all that that implies. Most training for work in a medical records department is by haphazard apprenticeship, though there are honourable exceptions-for example, at St. Bartholomew's Hospital, in Bristol, and in Scotland. But most of the full-time students come from overseas. The profession must be made attractive here at home.

One cannot but feel deep sympathy for the Secretary of State; his predecessors concerned with health alone had plenty to occupy them. The permanent staff of the Department of Health are busy enough too. Among many other things they are faced with demands, each of which considered in isolation is reasonable enough, for new kinds of medical auxiliaries. And the Health Service has turned out to be extremely costly; but it is the increasing complexity of medicine that has created these demands.

What if anything should be done about the people we call medical records officers (now due for a change of name, like the former lady almoners)? They can go on beating their own drum; loud bangs instead of a demure tattoo. The effectiveness of this is limited. The second, and the best, is for the Department to establish the medical records officers as a profession ancillary to medicine with all that that implies. Otherwise only one course remains-for us, the medical profession, who write the records and guard the confidences of our patients, to make such a rumpus that the Secretary of State will listen and act; so that in quietness and confidence we may write the truth, the whole truth, and nothing but the truth and make the best possible use of it.

\section{References}

1 Bunker, J. P., Pharos, 1971, 34, 20.

2 Bunker, J. P., New England fournal of Medicine, 1970, 282, 135.

3 Baldwin, J. A., Proceedings of Second European Conference on Medical Records, 1970.

4 Pickering, G. W., British Medical fournal, 1971, 1, 191.

\title{
Problems of the Newborn
}

\section{Prenatal Influences and Prenatal Diagnosis}

\author{
R. W. SMITHELLS, B. D. SPEIDEL
}

\section{British Medical fournal, 1971, 4, 105-108}

Perinatal mortality has fallen dramatically since the turn of the century and is still falling. The present rate of 23 per 1,000 births is accounted for largely by babies of low birth weight, those with major congenital anomalies, and those subjected to severe hypoxia. Haemolytic disease and infections are responsible for a diminishing number of perinatal deaths. All \footnotetext{
Department of Paediatrics and Child Health, University of Leeds,
Leeds LS1 3ET

R. W. SMITHELLS, F.R.C.P., F.R.C.P.E., Professor of Paediatrics and

B. D. SPEIDEL, B.M., M.R.C.P., Tutor in Paediatrics
}

these conditions are capable of causing physical or mental handicap in survivors. Further progress can be made, firstly, by identifying and correcting any influences which are harmful to the developing fetus before and during birth and, secondly, by recognizing and treating disorders at the first possible moment. These require continuing research, the implementation of existing knowledge, and unceasing vigilance.

\section{Fetal Growth}

\section{PRENATAL INFLUENCES}

The relationship between low birth weight and maternal height, age, parity, and socio-economic status shows that maternal 
factors have an important influence on fetal growth. The effects of these factors are not limited to growth, perinatal mortality from all causes being increased among the offspring of short mothers, an undue proportion of whom come from social classes IV and V. Among other adverse factors, probably poor nutrition in childhood and pregnancy results in poor reproductive function, but maternal genetic factors are also concerned.

Diabetic and prediabetic women usually have large babies. Nevertheless, if the diabetes is severe the fetus may be malnourished, presumably because of vascular complications of the disease. Indeed, many cardiovascular disorders appear to cause placental insufficiency and hence retardation of fetal growth. For example, mothers with heart disease and renal or essential hypertension tend to have small babies. Similarly, severe or prolonged toxaemia may also be associated with retarded fetal growth.

It is not certain whether the harmful effects of smoking in pregnancy are mediated by vascular changes but it seems likely. What is certain is that smoking by the pregnant woman, even to a very modest extent, retards fetal growth and that this effect is independent of maternal age, height, and social class. Indeed, smoking increases the risks of miscarriage, stillbirth, and early neonatal death. ${ }^{1}$

In practical terms poor fetal growth can best be prevented by high standards of health throughout childhood and pregnancy.

\section{PRENATAL DIAGNOSIS}

Fetal growth must be monitored throughout pregnancy, especially when any of these adverse influences are present. If clinical assessment seems inadequate, special tests may be helpful.

There is no completely satisfactory way of assessing fetal maturity. The date of the mother's last menstrual period is a valuable guide, but is not always reliable, nor is assessment of the size of the fetus from the height of the uterine fundus very accurate, though serial observations are more informative. Radiological examination of fetal epiphyses shows better correlation with fetal maturity than do the mothers' dates, ${ }^{2}$ but this is not helpful in the diagnosis of retardation of intrauterine growth.

The biparietal diameter of the fetal skull may be measured by the use of ultrasound, and this correlates well with the length of gestation. S. Campbell ${ }^{3}$ used this method between the 20th and 30th weeks to measure the rate of growth of the fetal skull and was able to predict the day of delivery with remarkable accuracy. The technique has been -used to detect growth retardation successfully in $70 \%$ of cases. ${ }^{4}$

Both the fetus and placenta synthesize oestriol. This is excreted in maternal urine, where its concentration provides an index of placental function and of fetal health. Subnormal oestriol levels in serial samples of urine are useful in the diagnosis of retardation of fetal growth, and falling, low levels indicate impending fetal death and the need for urgent delivery.

Amniotic fluid may be obtained by amniocentesis from about the 14th week of pregnancy, provided proper precautions are taken. This fluid contains fetal urine and consequently its biochemical composition is influenced by the efficiency of the fetal kidney. The concentrations in the amniotic fluid of creatinine and urea rise, while its osmolality falls, as pregnancy progresses.

From the 30th week of pregnancy onwards the amniotic fluid contains increasing numbers of desquamated fetal cells. The percentage of these cells which are anucleate and stain orange with nile blue sulphate can be related to the length of gestation. ${ }^{5}$ By other staining techniques (Papanicolaou or haematoxylin and eosin) the cells may be differentiated into two types: basal and precornified. The basal cells disappear by the 35th week leaving the precornified cells, which gradually become cornified as term approaches. T. Lind and W. Billewicz ${ }^{6}$ have unified these amniotic fluid characteristics into a system for estimating gestational age which is within the capabilities of most hospital laboratories.

\section{Haemolytic Disease}

\section{PRENATAL INFLUENCES}

The introduction of anti-D globulin has revolutionized the management of Rhesus isoimmunization. It has not diminished the need to understand those conditions which predispose to feto-maternal bleeding and hence to the risk of maternal sensitization (the minimum amount of blood needed to cause sensitization is very small, possibly as little as $0.5 \mathrm{ml}$.) Information about obstetric influences on feto-maternal haemorrhage is conflicting, but probably any disturbance of the normal placento-uterine relationship may predispose towards such bleeding. An increased transfer of fetal red cells has been reported following caesarian section, forceps delivery, prolonged labour, the use of oxytocin, and manual removal of the placenta.

The rhesus antigen is present in the 6-week-old embryo: sensitization may therefore occur after spontaneous or therapeutic abortion. Though the risk of sensitisation after abortion is less than $1 \%$, the increasing use of this procedure makes it important that anti-D globulin should be given to all rhesusnegative women who abort as well as to those who go on to term. From 1 July 1971 anti-D globulin has been available in Britain for all rhesus-negative women without antibodies.

\section{PRENATAL DIAGNOSIS}

Routine rhesus grouping with subsequent screening of rhesusnegative women for the presence of antibodies remains the backbone of prenatal diagnosis, but with the increasing use of anti-D globulin the development of antibodies in a previously unsensitized woman should become rare. There remain those mothers who already have antibodies. In them neither the past history of affected infants nor the antibody titre gives a reliable indication of how severely the fetus is affected. The concentration of bilirubin in the amniotic fluid as measured by spectrophotometry shows a better correlation. Using Liley's ${ }^{7}$ method, the bilirubin level as calculated from optical density measurements at a wavelength of $450 \mathrm{~nm}$ is plotted against the length of gestation on a standard graph. The resulting curve gives a good idea of how severely the fetus is affected and can help to decide whether early delivery or intrauterine transfusion is required.

It should be emphasized that, though the D-antigen is concerned in $93 \%$ of cases of rhesus haemolytic disease, other rhesus, ABO, and rare antigens may be implicated and these are not excluded by routine prenatal tests.

\section{Fetal Hypoxia}

\section{PRENATAL INFLUENCES}

Perinatal asphyxia remains a potent cause of death and permanent brain damage; consequently it is important to be aware of the conditions in which it may occur. Antepartum hypoxia may be due to the placental insufficiency which accompanies pre-eclamptic toxaemia, prolonged gestation, and retro-placental bleeding. B. M. Hibbard ${ }^{8}$ has shown that abruption of the placenta is more likely to occur in mothers who are deficient in folic acid. Asphyxia may occur with prolonged labour, prolapse of the cord, and malpresentations. Heavy maternal sedation, particularly with opiates, may depress the baby's respiratory centre and so cause neonatal asphyxia. 


\section{PRENATAL DIAGNOSIS}

The value of urinary oestriol levels in the recognition of placental insufficiency has already been mentioned. The diagnosis of fetal distress during labour is usually based on the passage of meconium-stained liquor and an alteration in fetal heart rate outside certain limits. Nevertheless, only $15 \%$ of babies with clinical fetal distress are found to be asphyxiated at birth.

Meconium-staining of the liquor may be seen before the membranes have ruptured by amnioscopy. After their rupture, when the vertex is presenting, samples of fetal scalp blood can be taken and the $\mathrm{pH}, \mathrm{Po}_{2}$, and $\mathrm{PCO}_{2}$ measured, giving direct evidence of true asphyxia. ${ }^{2}$ This technique is most useful when combined with continuous monitoring of the fetal heart in high risk cases, by fetal electrocardiograph or by the cardiotochograph (which records both uterine contractions and fetal heart rate).

\section{Fetal Development}

\section{PRENATAL INFLUENCES}

Influences leading to fetal malformations include specific causes (of which very few are known) and predisposing factors. The latter include maternal age, parity, and social class, about which information is easy to collect but difficult to interpret. Genetic factors may be causal (as in single gene defects) or act as predisposing factors (as in cleft lip and palate). It is sometimes difficult to know whether geographical and racial variations are essentially genetic, environmental, or both. Studies of migrants and of children born to parents of mixed races may unravel these problems.

The effect of maternal age is most clearly seen in relation to Down's syndrome. Over half the affected infants are born to mothers aged 35 or more and about $30 \%$ to mothers in their 40 s. Less widely recognized is the rising incidence of haemophilia and achondroplasia with increasing paternal age.

Information about other factors which may contribute to the production of malformations has come from epidemiological studies, particularly of neural tube defects (anencephaly and spina bifida). These defects are more common at the extremes of maternal age and parity and are more prevalent in the lower socio-economic groups. They are also commoner in the north and west of Britain than in the east and south, a finding that parallels the socio-economic status of these regions and their underlying geological structure. Social class differences alone do not explain these geographical variations. ${ }^{10}$

\section{Fetal Compression}

Fetal compression within the uterus may possibly play a part in the aetiology of talipes, congenital dislocation of the hip, retrognathia (backward displacement of the jaw), and cleft palate. Almost certainly congenital amputations and ring constrictions may be caused by amniotic bands. ${ }^{11}$

Infectious agents may be transmitted across the placenta and harm the developing embryo or fetus. The disastrous results of rubella in early pregnancy are of particular importance because it is one of the few causes of severe malformations that is potentially preventable. Rubella may be mimicked by various non-teratogenic virus infections, including Coxsackie, ECHO, and adenoviruses. Nevertheless, maternal immunity to rubella may be demonstrated rapidly by the presence of haemagglutination inhibiting antibody. Effective rubella vaccines are available for girls aged 11 to 14 years and for selected susceptible women. Other infectious agents which are not teratogenic but which may cause fetal damage include Toxoplasma gondii, cytomegalovirus, and Herpesvirus hominis.

The incidence of malformations in the offspring of diabetic mothers seems to be higher than expected. L. M. Pedersen and his colleagues ${ }^{12}$ gave the overall risk of malformations as three times greater than normal and that for fatal and multiple anomalies as six times greater. J. W. Farquhar ${ }^{18}$ found a somewhat lower incidence but still greater than expected.

\section{Effects of Drugs}

The potential dangers of drug administration during pregnancy are well known. M. M. Nelson and J. O. Forfar, ${ }^{14}$ in a survey of the relation between drugs taken in pregnancy and congenital abnormalities, found that $97 \%$ of mothers had taken prescribed drugs and $65 \%$ had taken non-prescribed ones. Nevertheless, only a few drugs are definitely known to be teratogenic in man. Little need be said about thalidomide except for the reminder that extreme caution is needed in the use of new drugs in pregnancy. Cytotoxic drugs, some of which are used as immunosuppressive agents, may cause severe fetal damage, but will be used in pregnancy only when the need is unavoidable. There is a small risk that a cleft palate may follow the use of corticosteroids, though this does not apply when they are used for replacement therapy.

The effects of drugs on the fetus are not always teratogenic. Norethisterone, sometimes used in the management of recurrent abortion, may cause virilization of the female fetus. Other progestational agents appear to be safe in this respect. Antithyroid drugs given to the mother may cause fetal hypothyroidism, with or without goitre.

It is important to remember that drugs given in the later part of pregnancy may have undesirable side effects on the fetus. For example, tetracycline may discolour the teeth, streptomycin may cause deafness, and sulphonamides and salicylates may precipitate kernicterus.

\section{PRENATAL DIAGNOSIS}

Techniques for studying the fetus in utero are rapidly being developed, but their use is limited and they are employed only if the clinician is anxious about fetal development. This anxiety may be aroused by a family history of a hereditary disorder or a past history of recurrent abortion. Persistent malpresentation is rarely due to fetal anomaly, though babies with hydrocephalus or renal agenesis often present by the breech and anencephalics by the face.

Oligohydramnios is not easily recognized but is often associated with those anomalies of the fetal genitourinary tract that prevent the passage of urine into the amniotic cavity. Polyhydramnios, on the other hand, occurs in $1 \%$ of all pregnancies, and of these $25-40 \%$ are associated with a malformation. ${ }^{15}$ Anencephaly and iniencencephaly (a related malformation of the brain, base of the skull, and cervical) spine probably prevent the fetus swallowing amniotic fluid, and high intestinal obstructions prevent the swallowed fluid from being absorbed; hence these disorders may cause polyhydramnios.

\section{Radiography}

Radiography is most useful in the diagnosis of skeletal defects but special methods, such as amniography, are needed to demonstrate anomalies of the soft tissues. ${ }^{16}$ Amniography involves the injection of a radio-opaque medium into the amniotic fluid to outline the fetus and thereby to show up such malformations as meningomyelocele and encephalocele. The contrast medium is swallowed by the fetus, and malformations of the gastrointestinal tract may be shown.

The fetus can be examined directly by transabdominal fetoscopy and external malformations recognized, but this technique is still at a very early stage of development.

The adrenogenital syndrome may be diagnosed at about the 
37th week of pregnancy by demonstrating raised levels of 17-ketosteroids and pregnanetriol in amniotic fluid. In this condition, which may be difficult to diagnose at birth, there is a risk of sudden death in the early neonatal period and prenatal diagnosis may therefore be helpful.

\section{Determination of Fetal Sex}

Genetic diagnosis has extended into the prenatal period ${ }^{17}$ and it is now possible to determine fetal sex by finding Barr bodies or by showing fluorescence of the $\mathrm{Y}$ chromosome in amniotic fluid cells. This is most useful if the mother is a carrier of an $\mathrm{X}$-linked gene for serious disorders such as haemophilia or Duchenne muscular dystrophy when males carry a $50 \%$ risk of having the disease.

Amniotic cell culture may be used to demonstrate abnormal chromosomes. For example, there is a high risk of Down's syndrome in the offspring of $G / D$ translocation carriers. If the disorder is diagnosed in the fetus in early pregnancy, termination may be considered. Unfortunately, amniocentesis is not feasible before the 12th week of pregnancy and cell culture takes another four weeks, by which time pregnancy is well advanced. Nevertheless with further progress it may be possible to reduce the culture time.

Over 30 rare metabolic disorders can be recognized by biochemical study of cultured cells. The field is a rapidly expanding one and is increasingly being applied to prenatal diagnosis. Many of these disorders are inherited as recessives, when the recurrence risk is $25 \%$, but with these new techniques it should be possible in future to give a positive diagnosis with an accuracy approaching $100 \%$.

\section{References}

1 Royal College of Physicians of London, Smoking and Health Now. London,

Pussell, J. G. B., Fournal of Obstetrics and Gynaecology of the British Commonwealth, 1969, 76, 208.

3 Campbell, S., Fournal of Obstetrics and Gynaecology of the British Commonwealth, 1969, 76, 603 .

Willocks, J., Donald, I., Campbell, S., and Dunsmore, I. R., fournal of Obstetrics and Gynaecology of the British Commonwealth, 1967, 74, 639.

5 Brosens, I., and Gordon, H., Fournal of Obstetrics and Gynaecology of the British Commonwealth, 1966, 73, 88.

- Lind, T., and Billewicz, W., British fournal of Hospital Medicine, 1971, 5, 681.

' Liley, A. W., American Fournal of Obstetrics and Gynaecology, 1961, 82,

Hibbard, B. M., fournal of Obstetrics and Gynaecology of the British Commonwealth, 1964, 71, 529.

- Saling, E., Foetal and Neonatal Hypoxia in Relation to Clinical Obstetric

10 Fractice. London, Arnold, 1968. .

11 Fedrick, J., Annals of Human Genetics, 1970, 34, 31 . Gestation. Springfield, Illinois, Thomas, 1968.

12 Pedersen, L. M., Tygstrup, I., and Pedersen, J., Lancet, 1964, 1, 1124.

13 Farquhar, J. W., Archives of Disease in Childhood, 1969, 44, 36.

14 Nelson, M. M., and Forfar, J. O., British Medical fournal, $1971,1,523$.

Stevenson, A. C., in Ciba Foundation Symposium on Congenital Malformations, ed. G. E. W. Wolstenholme and C. M. O'Connor, p. 241. London, Churchill, 1960.

16 Queenan, J. T., and Gadow, E. C., Obstetrics and Gynecology, 1970, 35, 648.

17 Nadler, H. L. Fournal of Pediatrics, 1969, 74, 132.

\title{
Medical Education
}

\section{Medical Television in the Inner London Area}

\author{
MICHAEL CLARKE, IAN GILLILAND
}

\section{British Medical fournal, 1971, 4, 108-109}

The Inner London Education Authority has established a closed circuit television network in Inner London by means of a cable laid by and rented from the Post Office. Channel 7 of this network is for higher education. The University of London has developed an Audio-Visual Centre, which, as part of the work which the centre does for the many disciplines of London University, has prepared television recordings for postgraduate medical education. These programmes are produced in co-operation with the British Postgraduate Medical Federation and with financial support from the four metropolitan regional hospital boards. The medical programmes will be transmitted on Channel 7.

The University Audio-Visual Centre's headquarters are at 11 Bedf $\cap$ rd Square in London, and it is here that programmes are planned and produced. In this building there is a small studio for television recording and equipment for film making, including animated film, photography, sound recording, and

\section{University of London}

MICHAEL CLARKE, M.A., Director, University of London Audio-

Visual Centre
IAN GILLILAND, M.D., F.R.C.P., Assistant Director, British Postgraduate Medical Federation graphic design. Most of the medical programmes are made in the centre but, where necessary, a mobile unit can go outside to record material. This mobile unit is in a large van which contains a control room with three cameras and the necessary staff. Recordings can thus be made anywhere in the metropolitan area-in clinics, laboratories, theatres, or wayds. Film can also be made where extra precision and control are needed and the film subsequently included in a television recording.

The British Postgraduate Medical Federation, as part of the University of London, is responsible for medical postgraduate education in a large area of southern England which includes some 72 academic centres in district hospitals of the four metropolitan regional hospital boards. Soon the 12 undergraduate teaching hospitals, the 15 postgraduate institutes, and 11 of the major district hospitals in the Inner London area will be connected to the network, so that there is a large potential audience. In all these attached centres medical programmes can be seen by switching the receiver at the appropriate time to Channel 7 .

\section{Object of Programmes}

The object of these programmes is to enable as many medical personnel as possible to see and hear expert teachers who cannot visit all these centres in person, while at the same time providing the experts with all the aids which they might use in their usual teaching-graphs, diagrams, charts, photo- 